\title{
The green generation of sunscreens: Using coffee industrial sub-products
}

\author{
J. Marto ${ }^{\text {a }}$, L.F. Gouveia ${ }^{a}$, B.G. Chiari ${ }^{\mathrm{b}}$, A. Paiva ${ }^{\mathrm{c}}$, V. Isaac ${ }^{\mathrm{b}}$, P. Pinto ${ }^{\mathrm{a}}$, P. Simões ${ }^{\mathrm{c}}$, \\ A.J. Almeida ${ }^{a}$, H.M. Ribeiro ${ }^{a, *}$ \\ ${ }^{a}$ Research Institute for Medicines and Pharmaceutical Sciences (iMed.UL), Faculty of Pharmacy, University of Lisbon, Portugal

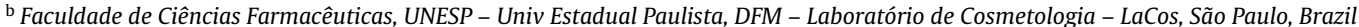 \\ c LAQV-REQUIMTE, Departamento de Química,Faculdade de Ciências e Tecnologia, Universidade Nova de Lisboa, Caparica, Portugal
}

\section{A R T I C L E I N F O}

\section{Article history:}

Received 6 July 2015

Received in revised form 4 November 2015

Accepted 9 November 2015

Available online 6 December 2015

\section{Keywords:}

Spent coffee oil

Green coffee oil

Supercritical fluid extraction

Water resistant

Pickering emulsion sunscreen

\begin{abstract}
A B S T R A C T
Spent coffee grounds and green coffee defective beans, which are industrial sub-products of coffee processing, have a potential use for cosmetic applications, due to their safety and high content in lipids that present interesting physicochemical properties. Sunscreen formulations in the form of water-in-oil emulsions might be a suitable application for these sub-products because providing a higher sun protection factor (SPF) for the same concentration of sunscreen activities than oil-in-water emulsions.

The purpose of this work was to assess the biological effects of using the oil fraction of spent coffee grounds extracted with supercritical $\mathrm{CO}_{2}$ and green coffee oil in the development of new generation of sunscreens with improved sun protection performance. The oil fractions were used to prepare w/o sunscreens involving a cold emulsification process, with purified water as disperse aqueous phase and $\mathrm{TiO}_{2}$ and $\mathrm{ZnO}$ particles as stabilizers. The sunscreens were characterized in terms of mechanical, rheological and skin adhesion properties. In addition, the in vitro and in vivo biological properties of the formulations were evaluated, including safety and sunscreen water resistance tests.

The use of two types of solid particles proved to be useful in the developed formulations, ensuring a high SPF with UVB/A protection, conferred by $\mathrm{TiO}_{2}$ and $\mathrm{ZnO}$, respectively. Moreover, the emulsion containing $35 \% \mathrm{w} / \mathrm{w}$ of the spent coffee grounds oil fraction presented promising characteristics in the improvement of water performance with a broad spectrum sun protection when compared to an emulsion containing 35\% w/w of green coffee oil which improved the SPF in physical sunscreens. The formulations are industrial-scalable and suitable for topical use according to the rheological, mechanical and safety assessment.

The use of spent coffee oil in cosmetic industry seems to be a suitable approach for the valorisation of waste from the coffee industry and presents promising characteristics in the improvement of sunscreen performance.
\end{abstract}

(c) 2015 Elsevier B.V. All rights reserved.

\section{Introduction}

The efficacy of sunscreen products has been recognized as an important public health issue and is usually expressed by the sun protection factor (SPF), which is calculated as the ratio between the UV energy required to produce a minimal erythema dose of protected and unprotected skin (Dutra et al., 2004; Ribeiro et al., 2013).

\footnotetext{
* Corresponding author at: Research Institute for Medicines (iMed.ULisboa), Fac ulty of Pharmacy, Universidade de Lisboa Av. Professor Gama Pinto, 1649-003 Lisboa, Portugal. Fax: +351 217946470.

E-mail address: hribeiro@campus.ul.pt (H.M. Ribeiro).
}

Avoiding sun exposure, covering the skin or applying sunscreens with a high SPF are the main strategies strongly recommended to prevent UV-induced cell damage. The UV filters can be divided in two groups: (a) chemical filters which absorb UV radiation (UVR); and (b) physical filters, such as, titanium dioxide $\left(\mathrm{TiO}_{2}\right)$ which reflect UVR (Ascenso et al., 2014).

Sunscreens are normally based on synthetic chemicals with high capacity to absorb sun light at the region of UVB (320-290 nm) and UVA (400-320 nm) spectrum. Several synthetic UV filter molecules (e.g. benzophenones, anthranilates, PABA derivatives, salicylates, cinnamates and camphor derivatives) are available as photoprotective agents, but due to their harmful effects they are becoming less popular. The main problem of the chemical sunscreen agents 
are the photoirritation, photosensitization and contact dermatitis. Reducing the concentration of such chemicals in cosmetics is a strategy to improve their quality, without affecting their properties (Serpone et al., 2007).

In recent years, the use of natural agents has been attracting significant attention, due to their safety, multiple biological actions on the skin and cost effectiveness. Consequently, an oily vehicle with antioxidant activity could be an excellent approach for its important role on the product efficacy by improving the products' photoprotective activity (Calixto et al., 2011; Couto et al., 2009). In addition to bioactivity, natural products are, in general, not harmful for humans, not expensive, suitable to be used in a wide range of applications, and are obtained from renewable sources. In this context, green coffee oil and spent coffee grounds oil have arisen as potential candidates to replace synthetic chemicals in sunscreens since they are a rich source of antioxidants and polyphenols (Ribeiro et al., 2013). Phenolic compounds are excellent candidates for the prevention of the harmful effects of UV radiation on the skin. More specifically, flavonoids have photoprotection potential due to their UV absorbing capacity, ability to act as antioxidants and anti-inflammatory, and immunomodulatory agents (Saewan and Jimtaisong, 2013).

Coffee consumption is growing worldwide, being extremely embedded in the cultural habits of many countries, so the total import and export of coffee has been increasing. Spent coffee grounds (SCG), which are the residue obtained after the treatment of coffee with hot water or steam for extracting flavour substances, can be used for industrial applications such as high quality biodiesel production (Couto et al., 2009). Nevertheless, due to their high content in lipids, particularly fatty acids, SCG might also find a suitable application in cosmetic products where these lipid compounds can be used as valuable excipients. Therefore, the lipid fraction of SCG extracted with supercritical $\mathrm{CO}_{2}$ can be used in the development of new, improved sunscreens. On average, a fifth of the Brazilian coffee production consists of defective beans, and several studies have been developed in order to find an alternative use for subproduct, including the cosmetic application of the extracted oil (Preedy, 2014; Ribeiro et al., 2013).

Titanium dioxide has been incorporated in sunscreen formulations for more than 25 years, being regarded as safe and effective, thus bringing together two of the most desirable features in cosmetic market (Renner, 2009). It is especially preferred by people with a high propensity for skin irritation, such as patients undergoing oncological chemotherapy. Moreover, $\mathrm{TiO}_{2}$ particles are a UV-B filter, suitable for developing physical sunscreens combining both increasing stability and high SPF properties (Wang and Tooley, 2011). The introduction of $\mathrm{ZnO}$ ensures an adequate protection in the range of UVA. Due to its multifunctional nature, $\mathrm{ZnO}$ particles have been shown to be effective as antibacterial and antifungal agent (Singh et al., 2012; Smijs and Pavel, 2011).

On the other hand, $\mathrm{TiO}_{2}$ particles can be also used as solid particles for stabilization of surfactant-free emulsions stabilized by solid particles (i.e. Pickering emulsions). This type of emulsions have important advantages over the classical surfactant-based emulsions, such as higher resistance to coalescence due to an improved stability, and a higher tolerability (Laredj-Bourezg et al., 2012). The stabilization of emulsion droplets takes place by means of adsorption of solid particles at the surface of emulsion droplets. It can be assumed that a stable water-in-oil (w/o) Pickering emulsion is a function of particles concentration, $\mathrm{pH}$ and ionic strength. This adsorption mechanism is quite different compared to surfactants since partial wetting of the solid particles surface by water and oil is the reason of the strong anchoring of these particles at the water-oil interface. Few fully natural and biocompatible materials are available for the effective stabilization of these emulsions since severe requirements must be simultaneously met, including insolubility in both fluid phases and intermediate wettability (Folter et al., 2012).

The purpose of this work was to develop and characterize a w/o emulsion stabilized by physical sunscreens containing $35 \%$ of the lipid fraction of spent coffee grounds extracted with supercritical $\mathrm{CO}_{2}$ and green coffee oil obtained from defective beans with improved sunscreen performance. Sunscreen formulations might be a suitable application for these types of sub-products because w/o emulsions are water resistant and provide greater efficacy (a higher SPF) for the same concentration of sunscreen actives than their o/w counterparts (Couteau et al., 2012).

\section{Material and methods}

\subsection{Materials}

Spent coffee grounds oil (SCO) was supplied by LAQVREQUIMTE - Departamento de Química, Faculdade de Ciências e Tecnologia (Caparica, Portugal). Green coffee oil (GCO) was supplied by Cooxupé - Cooperativa de Cafeicultores de Gauxupé, (Minas Gerais, Brazil). Triethoxycaprylylsilane titanium dioxide $\left(\mathrm{mTiO}_{2}\right)($ Unipure White LC 987$)$ was a gift from Sensient (Milwaukee, USA). The starch used was aluminum starch octenylsuccinate (ASt) (DryFlo ${ }^{\circledR}$ Plus) obtained from AkzoNobel (Amsterdam, Netherlands). Zinc oxide (ZnO) (Tego ${ }^{\circledR}$ Sun Z 500) was obtained from Evonik Industries AG (Essen, Germany). Purified water was obtained by inverse osmosis (Millipore, Elix ${ }^{\circledR} 3$ ).

\subsection{Methods}

\subsubsection{Characterisation of the formulation ingredients}

2.2.1.1. Wettability measurements. Contact angles of water, green coffee oil and spent coffee oil on $\mathrm{ZnO}, \mathrm{mTiO}_{2}$ and ASt in air atmosphere were measured at room temperature by using ConAnXL-a Microsoft Excel based workbook and add-in software (freely available upon request) as described in detail elsewhere (Marto et al., 2015). All measurements were performed in triplicate.

2.2.1.2. Particle size distribution. Particle size distribution was determined using a Malvern Mastersizer 2000 (Malvern Instruments, UK), coupled with a Hydro S accessory. Data were expressed in terms of relative distribution of volume of particles in the range of size classes, and given as diameter values corresponding to percentiles of 10,50 and 90 . The Span value is a useful parameter to characterize the particle size distribution broadness.

\subsubsection{Natural oils.}

2.2.1.3.1. Oil extraction. SCG oil was obtained by supercritical $\mathrm{CO}_{2}$ extraction as described elsewhere (Ribeiro et al., 2013). Supercritical fluid extraction can be an environmentally friendly alternative to traditional organic solvent extraction processes whereby extraction/separate recovery of oil and bioactive compounds from agro-industrial residues can be done without their degradation (Brunner, 2013). Mass transport is highly facilitated owing to favourable transport properties (high mass and thermal diffusivities coupled with low viscosities) and the solvation capacity of the supercritical fluid is tuneable by changing the operating conditions of pressure and temperature. The most commonly used supercritical fluid, carbon dioxide, is non-toxic, non-flammable, non-corrosive, relatively inert from a chemical point of view and environmentally friendly. Its relatively low critical temperature (304.3 K) allows extraction of thermolabile substances without degradation.

The supercritical $\mathrm{CO}_{2}$ extraction of SCG oil was done in a high pressure extraction pilot unit. SCG were first dried in an oven at 
Table 1

Qualitative and quantitative composition of the sunscreen formulations.

\begin{tabular}{lll}
\hline Ingredients & \multicolumn{2}{l}{ Quantitative composition (\%,w/w) } \\
\cline { 2 - 3 } & GCO sunscreen & SCO sunscreen \\
\hline Phase A & 35 & - \\
$\quad$ Green coffee oil & - & 35 \\
Spent coffee oil & & \\
Phase B & 20 & 20 \\
Triethoxycaprylylsilane titanium dioxide & 15 & 15 \\
Zinc oxide & 5 & 5 \\
Aluminum starch octenylsuccinate & & \\
Phase C & 25 & 25 \\
$\quad$ Purified water & & \\
\hline
\end{tabular}

$378 \mathrm{~K}$ with air circulation until a moisture value of about $1 \%$. Supercritical $\mathrm{CO}_{2}$ extractions were carried out at $55^{\circ} \mathrm{C}$ and 250 bar using $0.5 \mathrm{~kg}$ of dry SCG per batch with an average $\mathrm{CO}_{2}$ flowrate of $15 \mathrm{~kg} / \mathrm{h}$ for ca. $1 \mathrm{~h}$ of extraction.

GCO was obtained by cold pressed extraction. Briefly, green coffee beans were pressed and come back to be pressed again for a further three times and then the oil is bottled with nitrogen.

2.2.1.3.2. SPF measurement. GCO and SCO were accurately weighed $(0.25 \mathrm{~g})$, diluted with ethanol, followed by ultrasonication for $5 \mathrm{~min}$ and filtered through filter paper (Whatman ${ }^{\mathrm{TM}} 42$ ). The absorption spectra of samples solution were obtained in the range of 290-320 nm (Hitachi U-2001, USA) every $5 \mathrm{~nm}$, using a standard $1 \mathrm{~cm}$ quartz cell, and ethanol as the blank. Triplicates were made, followed by the application of the Mansur equation (Eq. (1)).

$\mathrm{SPF}_{\text {spectrophotometric }}=\mathrm{CF} \times \Sigma_{290}^{320} \mathrm{EE}(\lambda) \times \mathrm{I}(\lambda) \times \operatorname{Abs}(\lambda)$

where $E E(\lambda)$ is the erythemal effect spectrum; I $(\lambda)$ is the solar intensity spectrum; $\operatorname{Abs}(\lambda)$ is the absorbance of sunscreen product; $C F$ is the correction factor $(=10)$ (Mansur et al., 1986). The values of $E E \times$ I are constants determined by Sayre et al. (1979).

\subsubsection{Sunscreen formulations-green coffee oil sunscreen (GCO sunscreen) and spent coffee oil sunscreen (SCO sunscreen)}

Two formulations were developed (Table 1) based on their macroscopic appearance, physical stability and SPF value. The disperse aqueous phase was composed of purified water, the continuous oil phase consisted of GCO or SCO. Solid particles $\left(\mathrm{mTiO}_{2}\right.$, $\mathrm{ZnO}$ and ASt) were firstly dispersed in the oil phase. The oil and aqueous phases were then mixed using a high-speed homogenizer (UltraTurrax ${ }^{\circledR}$, IKA-Werke GmbH \& Co., KG, Germany) at room temperature (cold process).

\subsubsection{Characterization of the sunscreen formulations}

2.2.3.1. Droplet size distribution. The emulsions were examined 1 day after preparation, using an optical microscope (Olympus CX40, Japan) equipped with a video camera. The droplet size was determined using the image analysis software Olympus Stream Essentials ${ }^{\circledR}$. The size data was expressed in terms of relative size distribution of particles (BS, 1993).

\subsubsection{Structural analysis of the sunscreen formulations.}

2.2.3.2.1. Rheology studies. Shear rate $v s$ shear stress measurements were performed at $25^{\circ} \mathrm{C}$ using a HAAKE RS- 1 Rheometer, equipped with automatic gap setting (Thermo Scientific ${ }^{\mathrm{TM}}$, Waltham, USA). Rotational viscosity was determined using a C $35 \mathrm{~mm}$ cone geometry, with an angle of $2^{\circ}$. Dynamic or shear viscosity measurements were carried out by rotational shear experiments, between 1 and $1000 \mathrm{~Pa}$. Oscillation sweep tests were performed at frequencies ranging between 0.1 and $100 \mathrm{~Hz}$. The creep and recovery test was carried out with a shear stress of $1 \mathrm{~Pa}$ for emulsions, allowing $360 \mathrm{~s}$ for creep and $360 \mathrm{~s}$ for relaxation. All tests were performed on samples of about $1 \mathrm{~g}$, at $25 \pm 0.5^{\circ} \mathrm{C}$. All experiments were performed in triplicate using new samples for each measurement.

2.2.3.2.2. Texture profile analysis (TPA). A Texture Analyzer TA.XT Plus with Exponent 3.0.5.0 software (Stable Micro Systems Ltd., Godalming, UK) was used to examine hardness, elasticity, compressibility, adhesiveness and cohesiveness of the emulsion. A probe $(\mathrm{P} / 10,10 \mathrm{~mm}$ Delrin), which was twice depressed into the sample at a defined rate $(5 \mathrm{~mm} / \mathrm{s})$ to a desired depth $(15 \mathrm{~mm})$, allowing $15 \mathrm{~s}$ of delay between consecutive compressions. Six replicates were performed at $25^{\circ} \mathrm{C}$ for each formulation.

2.2.3.3. In vitro SPF. The SPF was assessed using the Optometrics SPF-290S Analyzer (Optometrics Corporation, Essex, UK). The samples were prepared by spreading $110 \mathrm{mg}$ of each formulation over a Transpore ${ }^{\circledR}$ tape $(70.7 \times 70.7 \mathrm{~mm})$ to obtain a film of $2 \mathrm{mg} / \mathrm{cm}^{2}$, as specified by the European Regulation (EC, 2009). Each sample was exposed to a xenon arc solar simulator, and the analyser performed scans in 6 different spots on the Transpore ${ }^{\circledR}$ tape substrate. Each scan takes a transmittance $(T)$ measurement every $2 \mathrm{~nm}$ from a wavelength ranging from 290 to $400 \mathrm{~nm}$. The Monochromatic Protection Factor (MPF) was determined for the selected wavelengths using Eq. (2). The SPF value was calculated using Eq. (3).

$\mathrm{MPF}=\frac{1}{T}$

$\mathrm{SPF}=\frac{\sum_{290}^{400} E \lambda . B \lambda}{\sum_{290}^{400} \frac{E \lambda . B \lambda}{M P F \lambda}}$

where $(E)$ is the spectral irradiance of terrestrial sunlight under controlled conditions and $(B)$ is the erythema effectiveness (Kale et al., 2010).

2.2.3.4. In vitro sun product water resistance. The water resistance of developed sunscreens was measured using an improved in vitro bath system. An amount of $2 \mathrm{mg} / \mathrm{cm}^{2}$ of sunscreen formulation was dispensed onto the plate, and carefully applied with a rubbergloved finger. After drying for $15 \mathrm{~min}$, the SPF of each sample was determined using the SPF 290 analyzer (Optometrics SPF-290S Analyzer). The samples were immersed in the in vitro bath system $\left(29 \pm 2^{\circ} \mathrm{C}\right)$ and washed away by the water flow $(150 \mathrm{rpm})$ during $20 \mathrm{~min}$. The samples were allowed to air dry for $15 \mathrm{~min}$ and SPF was measured again. The samples were immersed once more and washed during $20 \mathrm{~min}$. The samples were allowed to air dry for $15 \mathrm{~min}$ and SPF was measured to calculate the water resistance retention (\%WRR) of the sunscreens, as defined by Eq. (4).

$\% \mathrm{WRR}=\frac{\mathrm{SPF}_{\mathrm{wet}}}{\mathrm{SPF}_{\mathrm{dry}}} \times 100$

where $\mathrm{SPF}_{\text {dry }}$ and $\mathrm{SPF}_{\text {wet }}$ are the SPFs before and after water immersion, respectively (Ahn et al., 2008; COLIPA, 2005).

2.2.3.5. Skin adhesion properties. Skin adhesion measurements were performed using Texture Analyzer TA.XT Plus (Stable Micro Systems Ltd., Surrey, UK) equipped with a load cell of $5 \mathrm{~kg}$, cylinder probe of $10 \mathrm{~mm}(P / 10,10 \mathrm{~mm}$ Delrin) and a measuring system A/MUC (skin adhesion test rig), which holds the human skin. An amount of $2 \mathrm{mg} / \mathrm{cm}^{2}$ of each sample was applied onto the skin. The probe was immersed in an in vitro bath system $\left(29 \pm 2^{\circ} \mathrm{C}\right)$ and washed away by the flow of water $(150 \mathrm{rpm})$ during $40 \mathrm{~min}$, in order to simulate the water resistance method for sun protection products (COLIPA, 2005). The area under the curve (AUC) was calculated from the force-distance plot as the work of skin adhesion per square centimetre. The formulation given below was used to calcu- 
late the work of skin adhesion per square centimetre $\left(\mathrm{mJ} / \mathrm{cm}^{2}-\mathrm{Eq}\right.$. (7)). Each experiment was carried out in triplicate.

Workofskinadhesion $\left(\mathrm{mJ} / \mathrm{cm}^{2}\right)=\frac{\text { AUC }}{\pi r^{2}}$

whereby $\pi r^{2}$ is the surface area of the human skin that is in contact with the sunscreen formulations.

2.2.3.6. Human repeat insult patch test (HRIPT). A safety evaluation study was performed on emulsions, using the Marzulli and Maibach (1976) HRIPT protocol. In brief, the product was applied on the back of 51 healthy volunteers that gave their prior informed written consent. For the induction period, a series of nine patches (Finn Chamber standard) were performed over a period of 3 weeks. Reactions after patching were scored according to International Contact Dermatitis Research Group (Fregert and Bandmann, 1975). A 2 weeks rest period was maintained without application of the test material. During the challenge period, new patches were prepared and fixed in the same manner as in the induction period, but also on the right side of the back (i.e. a virgin site).

The protocol was approved by the local Ethical Committee and respected the Helsinki Declaration and the AFSSAPS regulations on performed HRIPT studies on cosmetic products. The study was conducted under the supervision of a dermatologist who participated in the evaluation of irritation/allergic reactions to the emulsions.

2.2.3.7. In vivo sun product water resistance. The water resistance of sunscreens was tested on 3 subjects (Fitzpatrick skin type II). Panellists cleanse their forearms using a mild cleanser and leave them to air dry for $30 \mathrm{~min}$ before starting the test. Initial cross-polarized images were taken after the sunscreens application $\left(2 \mathrm{mg} / \mathrm{cm}^{2}\right)$ on the inner forearm $\left(4 \mathrm{~cm}^{2}\right)$.

The amount of each sunscreen formulation left before and after water bath immersion was quantified via cross polarized imaging by means of the Visia ${ }^{\circledR}$ CA (Canfield Scientific, Faitfield, NJ). Panellists immerse their forearms into a water bath system $\left(29 \pm 2{ }^{\circ} \mathrm{C}\right)$ and washed away by the flow of water $(150 \mathrm{rpm})$ during $40 \mathrm{~min}$ (Ahn et al., 2008).

The RGB colour space of the raw bitmap images was converted to relative luminance using Image ${ }^{\circledR}$. From these images, average $L$ changes for each sunscreen area were obtained from histograms. Skin whiteness was defined as the change in $L$ value before and after water immersion, and the percentage of water resistance retention (\%WRR) of the sunscreens was determined according to Eq. (8).

$\% \mathrm{WRR}=\frac{\text { Lwashedprotector }- \text { Lskin }}{\text { Lprotector }- \text { Lskin }}$

\subsubsection{Statistical analysis}

The data are expressed as mean and standard deviation (mean $\pm S D$ ) of experiments. Statistical evaluation of data was performed using one-way analysis of variance (ANOVA). Tukey-Kramer multiple comparison test (GraphPad PRISM 5 software, USA) was used to assess the significance of the difference between the groups $(p<0.05)$.

\section{Results and discussion}

\subsection{Characterisation of the formulation ingredients}

\subsubsection{Wettability measurements}

In the surfactant-free system stabilized by solid particles, these will be preferentially wet by one of the liquids, with the more poorly wetting liquid becoming the disperse phase. Particle wettability at the oil-water interface is quantified by the contact angle, $\theta$, that
Table 2

Contact angle of water, GCO and $\mathrm{SCO}$ on $\mathrm{mTiO}_{2}, \mathrm{ZnO}$ and ASt (mean $\pm \mathrm{SD}, n=3$ ).

\begin{tabular}{lllc}
\hline \multicolumn{3}{l}{ Contact angle $\left(^{\circ}\right)$} & \\
\hline Samples & Water & Green coffee oil & Spent coffee oil \\
\hline $\mathrm{mTiO}_{2}$ & $106.5 \pm 0.7$ & $60.1 \pm 1.9$ & $9.7 \pm 3.3$ \\
$\mathrm{ZnO}$ & $100.2 \pm 2.6$ & $23.4 \pm 0.1$ & $13.1 \pm 2.5$ \\
$\mathrm{ASt}$ & $109.0 \pm 0.4$ & $10.6 \pm 2.3$ & $13.3 \pm 4.0$ \\
\hline
\end{tabular}

the particle makes with it, which will determine the type of emulsion. Generally, if the water contact angle is $<90^{\circ}$, the solid surface is considered hydrophilic and if the water contact angle is $>90^{\circ}$, the solid surface is considered hydrophobic. Concerning the emulsions stabilized by solid particles, if the contact angle, measured through the aqueous phase, is $<90^{\circ}$ the emulsion will be o/w and, by contrast, if the contact angle is $>90^{\circ}$ the emulsion will be w/o (Marku et al., 2012). In this study, $\mathrm{mTiO}_{2}, \mathrm{ZnO}$ and ASt will stabilized more effectively w/o emulsions. All solid particles have a contact angle with water $>90^{\circ}$, due to hydrophobic coatings, and simultaneously, a contact angle with Green coffee oil (GCO) and Spent coffee oil (SCO) $<90^{\circ}$ (Table 2). Thus, by combining these three particles it is possible to obtain a stable w/o emulsion.

\subsubsection{Particle size distribution}

Particle size distributions of $\mathrm{mTiO}_{2}, \mathrm{ZnO}$ and ASt showed that all particles were larger than $100 \mathrm{~nm}$ (Table 3), thus complying with the international "green" standards, whereby nanomaterials must be avoided (COSMOS, 2013).

\subsubsection{Natural oils}

3.1.3.1. SPF measurement. Several studies have been developed in order to find an alternative use for these defective coffee beans or spent coffee ground, involving the characterization of their lipid fractions. The GCO was extracted using mechanical pressing, whereas the lipid fraction of SCG was extracted with supercritical $\mathrm{CO}_{2}$, an environmentally friendly solvent that allows the extraction and recovery of the oil at such conditions that no degradation of the lipid composition oil occurs while avoiding the use of hazardous organic solvents (Ribeiro et al., 2013).

The GCO and SCO have potential application in health care products, including sunscreen formulations (Chiari et al., 2014). In particular, due to the richness of polyphenols and flavonoids, the pure GCO shows a SPF higher than 5, improving SPF and, consequently, decreasing the concentration of chemical and/or physical sunscreens in such formulations (Wagemaker et al., 2011). In this study, GCO presented an SPF value of $5.03 \pm 0.23$ while SCO presented only $1.57 \pm 0.07$, which is in line with the fact that the coffee roasting process diminishes the content of polyphenolic compounds (Dai and Mumper, 2010; Speer and Kölling-Speer, 2006).

\subsection{Characterization of the sunscreen formulations}

\subsubsection{Droplet size distribution}

The use of a surfactant-free emulsion might be a strategy to avoid skin irritations associated to this type of excipients (Marku et al., 2012). In Pickering emulsions the droplet is stabilized by a

Table 3

Particle size distribution of the different solid particles proposed (mean $\pm S D, n=6$ ).

\begin{tabular}{lrlrr}
\hline Solid Particles & \multicolumn{4}{l}{ Particle size distribution $(\mu \mathrm{m})$} \\
\cline { 2 - 5 } & \multicolumn{1}{l}{ Span } & \multicolumn{1}{l}{$\mathrm{d}(0.1)$} & $\mathrm{d}(0.5)$ & \multicolumn{1}{c}{$\mathrm{d}(0.9)$} \\
\cline { 2 - 5 } & & & & \\
$\mathrm{mTiO}_{2}$ & $37.36 \pm 1.38$ & $0.14 \pm 0.01$ & $0.19 \pm 0.01$ & $7.12 \pm 0.30$ \\
$\mathrm{ZnO}$ & $19.51 \pm 5.53$ & $0.16 \pm 0.01$ & $0.57 \pm 0.03$ & $11.38 \pm 3.74$ \\
$\mathrm{ASt}$ & $1.00 \pm 0.01$ & $7.28 \pm 0.01$ & $13.52 \pm 0.01$ & $20.82 \pm 0.01$ \\
\hline
\end{tabular}


Table 4

Droplet size distribution of GCO and SCO sunscreen (mean \pm SD, $n=625$ ).

\begin{tabular}{llllr}
\hline \multirow{2}{*}{ Formulation } & \multicolumn{4}{l}{ Droplet size distribution $(\mu \mathrm{m})$} \\
\cline { 2 - 5 } & Span & $\mathrm{d}(0.1)$ & $\mathrm{d}(0.5)$ & $\mathrm{d}(0.9)$ \\
\hline GCO sunscreen & $1.17 \pm 0.02$ & $3.01 \pm 0.11$ & $5.54 \pm 0.28$ & $9.49 \pm 0.55$ \\
SCO sunscreen & $0.90 \pm 0.03$ & $5.72 \pm 0.10$ & $8.87 \pm 0.13$ & $13.71 \pm 0.34$ \\
\hline
\end{tabular}

reduction of the bare oil-water interface by adsorption of small particles. The parameter to describe Pickering emulsions stability is the contact angle of the adsorbed particles, as explained above. Initially, a contact angle greater than $90^{\circ}$ is required in order to obtain a w/o emulsion. In this assay we evaluated the influence of the oil on Pickering emulsions droplet size distribution. Both sunscreen formulations showed a narrow droplet size distribution with a span value in the range of 0.9-1.2. The GCO sunscreen exhibited lower mean droplet size than SCO sunscreen (Table 4). Previous studies demonstrated that coffee extracts have extremely strong antioxidant properties than many other food products. This applies to both roasted and green coffee, although, the latter contains even ten times higher concentration of polyphenols (Budryn et al., 2013). Other study demonstrated that several polyphenols, could be adsorbed into the oil-water interface, present as waterand oil-insoluble particles and decrease the surface tension, providing very good stabilization of emulsions and, consequently, leading a decrease in droplet size (Luo et al., 2011; Wagemaker et al., 2011).

\subsubsection{Structural analysis of the sunscreen formulations}

3.2.2.1. Rheology studies. The flow curves (Fig. 1(a)) showed that the emulsions presented a shear-thinning and rheopetic behaviour. This behaviour could influence the sunscreen performance, creating a uniform, impenetrable and protective semi-solid film over the skin surface that is required to obtain an effective sunscreen.

In the range of $0-5 \mathrm{~Pa}$, both emulsions were not disrupted (Fig. 1(b and c). Thus, the values of $G$ ' and $G$ "' remained linear within this region of linear viscoelasticity, which can therefore indicate the suitable shear stress to be used in frequency sweep and creep and recovery tests.

The GCO sunscreen exhibited higher $G$ ' and $G$ " values than SCO sunscreen. The frequency sweep curves of GCO sunscreen (Fig. 1(b)) showed that in the range tested $(0.1-100 \mathrm{~Hz})$ there was practically no variation in the elastic and viscous moduli. Furthermore, viscoelastic behaviour was seen over the whole range, since $G$ ' (elastic modulus) was higher than $G^{\prime \prime}$ (viscous modulus). The same relation is observed for SCO sunscreen, but the $G$ ' and $G$ " values for GCO sunscreen are higher than for SCO sunscreen, exhibiting high stability.

In the creep and recovery test, both sunscreens suffered deformation, shown by the compliance value $(J)$, but SCO sunscreen was much more susceptible to this force (Fig. 1(c). In the recovery part of this assay, when the shear stress was removed and the samples could recover their former structure, the elastic part of the deformation, was reversed.

In summary, the addition of GCO promoted an increase in the apparent viscosity and the elastic modulus, when compared to the formulation prepared with SCO. Considering the results of the particle size distribution (Table 4), the reduction in droplet size results in an increase in the viscosity and storage modulus of the emulsions and the shear-thinning behaviour becomes even stronger, which suggested an enhancement in emulsion stability and performance (Pal, 1996).

3.2.2.2. TPA. The results of the calculations of textural parameters are collected in Table 5 . The adhesiveness is the only parameter, which differs between the two formulations $(p<0.05)$. Concerning the emulsions adhesiveness, which is more a surface characteristic
Table 5

Mechanical properties of the sunscreens extracted from the TPA mode (mean \pm SD, $n=3)$

\begin{tabular}{lclcl}
\hline Formulations & Hardness g & $\begin{array}{l}\text { Adhesiveness } \\
(|\mathrm{g} \mathrm{s}|)\end{array}$ & Cohesiveness & $\begin{array}{l}\text { Compressibility } \\
(\mathrm{g} \mathrm{s})\end{array}$ \\
\hline GCO sunscreen & $23.62 \pm 1.76$ & $39.76 \pm 0.27$ & $0.82 \pm 0.04$ & $29.87 \pm 1.91$ \\
SCO sunscreen & $25.23 \pm 2.26$ & $48.65 \pm 0.53$ & $0.83 \pm 0.01$ & $31.09 \pm 1.86$ \\
\hline
\end{tabular}

and depends on a combined effect of adhesive and cohesive forces, the presence of SCO caused an increase in this parameter.

According to Alves et al. (2003), during coffee roasting process there are changes in the fatty acid composition, increasing the trans-fatty acid levels, which increase the hydrophobic character of the final formulation and, consequently enhance the adhesive forces and water repellent performance (Shyr and Ou-Yang, 2015). Other authors proved that spent coffee oil also contains more hydrophobic compounds, such as fatty acids, fatty acid esters, medium-chain paraffins and olefins than green coffee oil or other vegetable oils (Kelkar et al., 2015).

Considering the hardness, cohesiveness and compressibility results, the lipid type did not influence these parameters.

The fatty acid tails are hydrophobic because they are non polar and the heads are hydrophobic because they are polar.

\subsubsection{In vitro $S P F$}

Both emulsions showed high values of SPF with a suitable UVA/UVB ratio (Table 6). For GCO there was a synergistic increase of SPF value (around 1.6 fold) when combined with physical filters as $\mathrm{mTiO}_{2}$ in GCO sunscreen. According to Wagemaker et al. (2011) the oil extracted from unroasted beans is used by the cosmetics industry due to its excellent properties for the human skin, particularly maintaining skin-moisture due to its fatty acid composition. In addition, its ability to absorb UV radiation in the UVB range allows its use in sunscreens as a SPF enhancer, allowing to reduce the amount of physical and chemical filters (Chiari et al., 2014).

\subsubsection{In vitro sun product water resistance}

The in vitro method measures the SPF following a defined water immersion procedure. For a product to be considered water resistant, the value for the lower $90 \%$ one-sided confidence limit has to be greater than or equal to $50 \%$. A 'waterproof' product should have a \%WRR exceeding $80 \%$ after two immersions, while the \%WRR of a 'water resistant' product should be more than 50\% (COLIPA, 2005).

After the first immersion, the GCO sunscreen presented a \%WRR of 62.6, which decreased in the second immersion to 50.7\%. In turn, SCO sunscreen showed a value of $83.1 \%$ at first immersion and a value of $80.8 \%$ of WRR after the second immersion. Based on this \%WRR, it is possible to ensure the 'water resistance' mark for GCO sunscreen and the 'waterproof' claim for SCO sunscreen. As mentioned previously, during the coffee roasting process the fatty acid levels increase, enhancing the hydrophobicity of the SCO sunscreen and, consequently, improving the effectiveness of water resistance performance (Kelkar et al., 2015). Additionally, hydrophobic sunscreen formulations can also serve as additional water barriers to help mitigate the disruption in stratum corneum caused by constant exposure to water (Shyr and Ou-Yang, 2015). In water resistant products typically more polar lipophilic ingredients can be found. Conversely, waterproofing sunscreens should contain more nonpolar ingredients, which is the case of SCO (Couteau et al., 2012).

\subsubsection{Skin adhesion properties}

Table 7 shows the skin adhesion properties of the formulations. The force necessary to detach the GCO and SCO sunscreen samples from the skin was statistically higher than the one needed to detach 
(a)

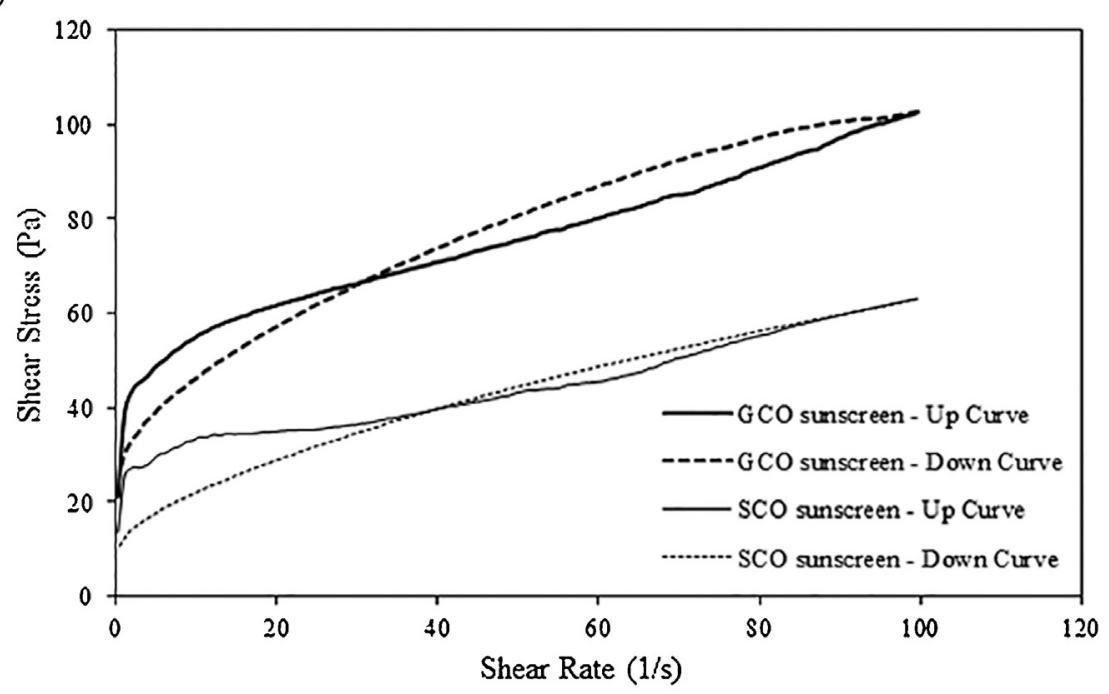

(b)

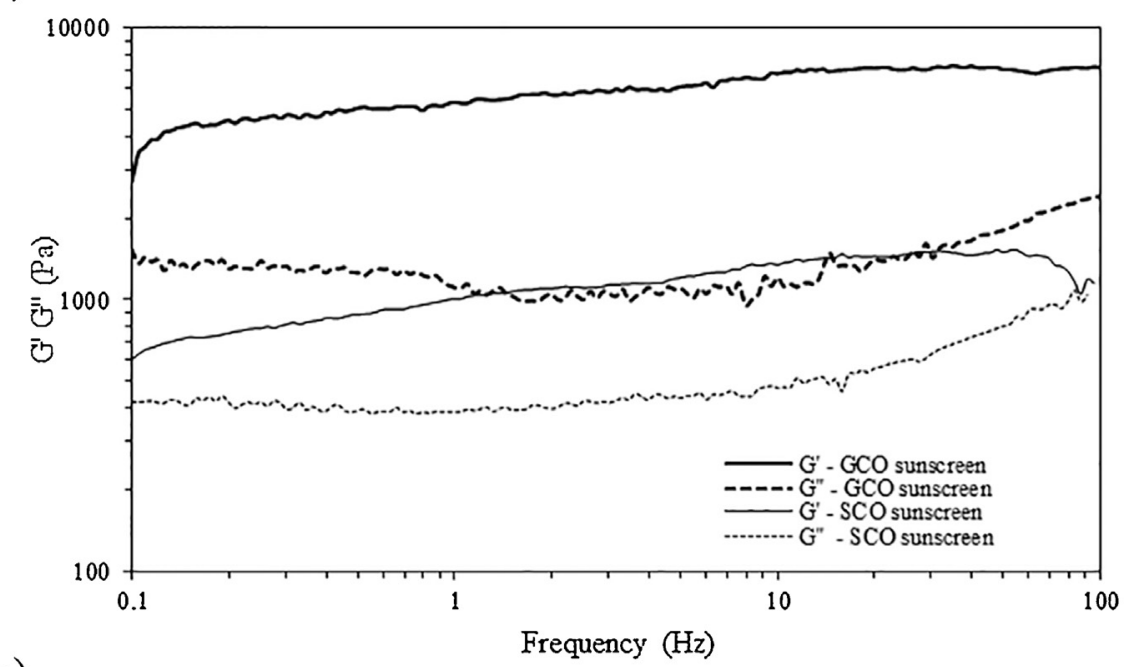

(c)

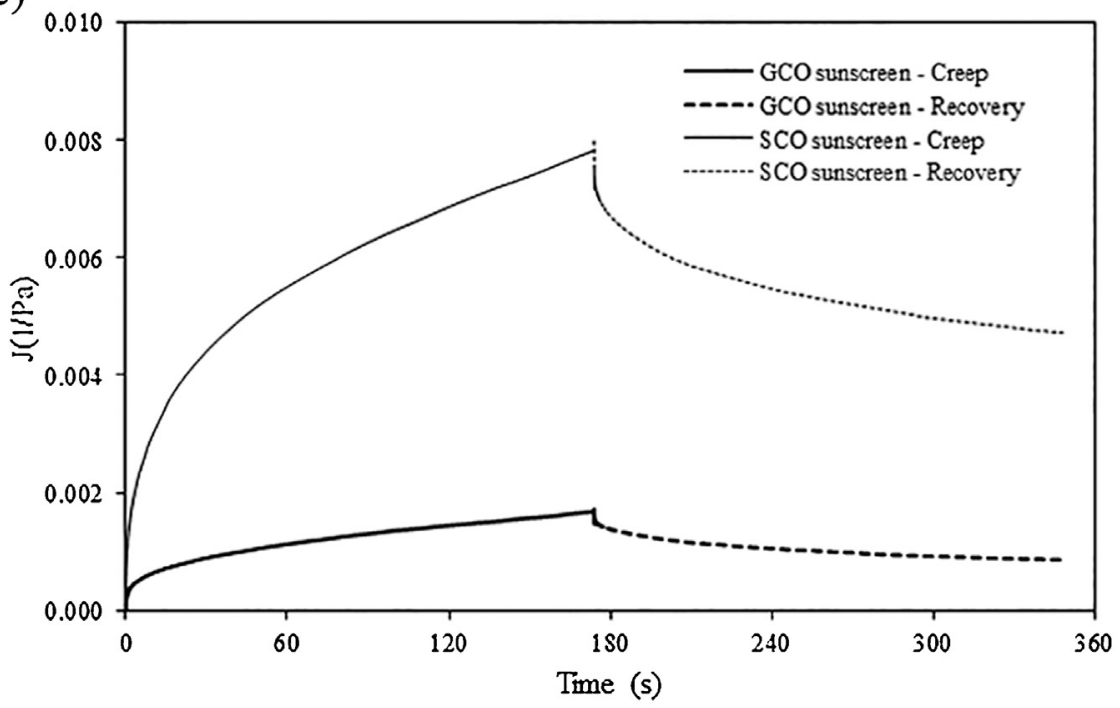

Fig. 1. (a) Flow curves, (b) frequency sweeps and (c) creep and recovery plots of GCO sunscreen and SCO sunscreen.

the blank. In our study, when the SCO was used, the work of skin adhesion ability after water immersion was also increased (Table 7). The type of lipid used in the preparation of water in oil emulsions sunscreens influenced the skin adhesion ability of emulsions and consequently the water resistant performance. According to the literature (Draelos, 2011), the lipid material used in the preparation 
Table 6

In vitro and in vivo efficacy tests of the GCO and SCO sunscreen.

\begin{tabular}{|c|c|c|c|c|c|c|c|c|}
\hline \multirow[t]{2}{*}{ Formulations } & \multicolumn{3}{|c|}{$\begin{array}{l}\text { In vitrosun protection factor } \\
\text { (SPF)-optometrics SPF-290S Analyzer }\end{array}$} & \multicolumn{4}{|c|}{ In vitro sun product water resistance } & \multirow{2}{*}{$\begin{array}{l}\text { In vivosun product } \\
\text { water resistance } \\
\text { \%WRR }\end{array}$} \\
\hline & Rating & UVA/UVB & UVA & $\begin{array}{l}\text { SPF after } \\
1 \text { st } \\
\text { immersion }\end{array}$ & $\begin{array}{l}\text { SPF after } \\
\text { 2nd } \\
\text { immersion }\end{array}$ & $\begin{array}{l}\% W_{R R}{ }^{a} \\
\text { after 1st } \\
\text { immersion }\end{array}$ & $\begin{array}{l}\text { \%WRR after } \\
\text { 2nd } \\
\text { immersion }\end{array}$ & \\
\hline GCO sunscreen & $82.3 \pm 1030+$ & $0.9 \pm 0.1$ & $71.1 \pm 10.1$ & $52.0 \pm 14.2$ & $42.1 \pm 13.4$ & 62.6 & 50.7 & $70.0 \pm 2.2$ \\
\hline SCO sunscreen & $51.9 \pm 5.550$ & $0.9 \pm 0.1$ & $44.2 \pm 5.2$ & $43.18 \pm 6.88$ & $41.95 \pm 5.94$ & 83.1 & 80.8 & $80.0 \pm 3.6$ \\
\hline
\end{tabular}

a Water resistance retention.

Table 7

Skin adhesive properties of the formulations on dry skin and after 40 min of water immersion (mean $\pm \operatorname{SD}, n=6$ ).

\begin{tabular}{|c|c|c|}
\hline Samples & Work of skin adhesion $\left(\mathrm{mJ} / \mathrm{cm}^{2}\right)$ & $\Delta$ of skin adhesion $\left(\mathrm{mJ} / \mathrm{cm}^{2}\right)^{\mathrm{a}}$ \\
\hline GCO sunscreen on dry skin & $0.05 \pm 0.01$ & - \\
\hline SCO sunscreen on dry skin & $0.05 \pm 0.01$ & - \\
\hline GCO sunscreen after water immersion & $0.01 \pm 0.01$ & 0.04 \\
\hline SCO sunscreen after water immersion & $0.04 \pm 0.01$ & 0.01 \\
\hline
\end{tabular}

a Work of skin adhesion between sunscreens on dry skin and after water immersion.
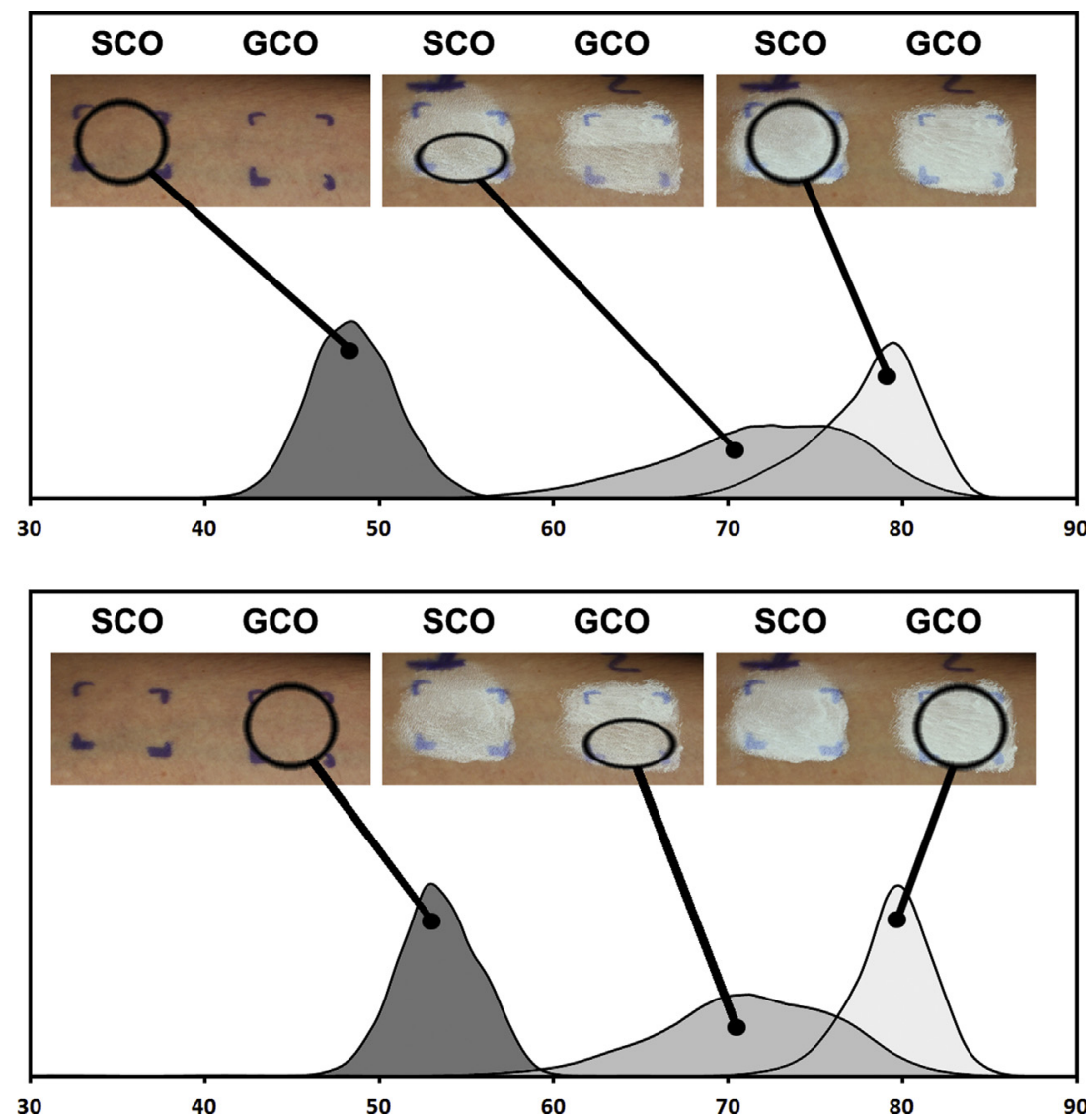

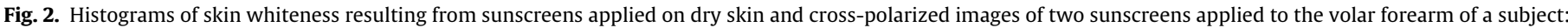
Dark grey-bare skin; grey-fresh sunscreen applications with 30 min air drying; and light grey - sunscreen after 40 min water immersion.

of w/o emulsions sunscreens influence the skin adhesion ability of emulsions and consequently their water resistance performance. The SCO extracted from roasted coffee is a brown viscous liquid due to the presence of liposoluble Maillard reaction products separated during the oil extraction, enhancing its lipophilic characteristics (Speer and Kölling-Speer, 2006). Concerning the skin surface properties, it was demonstrated that dry skin is mostly lipophilic and wet skin is more hydrophilic, having higher surface energy. Thus, the surface energy values of dry skin reflect its dominant lipophilicity and more apolar materials, such as SCO, may also adhere to skin.

\subsubsection{HRIPT}

Under the experimental conditions adopted, the repeated applications of the sunscreens under occlusive patch induced no irritative reaction and the product has very good skin compatibility. Moreover, the repeated applications induced no allergic reaction.

\subsubsection{In vivo sun product water resistance}

Human testing is considered to be the most acceptable method for claiming water resistance. This method is a new in vivo screening approach to measure WRR using cross-polarized imaging. Although it does not allow determining the exact SPF before and after the 
immersion, it allows evaluating the amount of sunscreen lost due to the action of water.

Skin whiteness results showed a similar behaviour as obtained for water resistance results (Fig. 2). After water immersion, both emulsions presented a range between 50 and $80 \%$ of whiteness with a mean peak around $70 \%$ and $75 \%$ of whiteness for GCO sunscreen and SCO sunscreen, respectively.

GCO sunscreen and SCO sunscreen, when exposed to water for $40 \mathrm{~min}$, revealed $70.0 \pm 2.2 \%$ and $80.0 \pm 3.6 \%$ of whiteness, respectively (Table 6). In vivo WRR values confirmed the results obtained in in vitro assay, thus supporting the "water resistant" claim of the GCO and SCO sunscreens. Furthermore, these in vivo values were higher than those obtained in vitro, which may be explained by a better adhesion to human skin compared with the adhesive tape used for the in vitro assay.

\section{Conclusions}

A novel sunscreen formulation with a high UVB/A protection, biological activity and better tolerability was developed based on the Pickering emulsions concept. The successful formulation was possible by combining natural and multifunctional compounds. Additionally, the coffee oils studied in this work possess all of the properties required for sunscreens and may be considered desirable and suitable ingredients for industrial applications. In addition to being a green product, it contains a series of lipophilic substances with important antioxidant characteristics, such as tocopherols, that protect the skin against UVB radiation.

When high oil contents are combined with a composition rich in unsaturated acids, and compounds with high SPF value and high unsaponifiable material content, the result is a product or ingredient that is ideally suited for formulating high quality cosmetic products able to promote moisture retention and simultaneously provide sun protection. All results revealed an excellent compromise between stability, UV protection, rheological and mechanical behaviour, efficacy, safety and cosmeticity.

The use of spent and green coffee oil in cosmetic industry seems to be a suitable approach to recycle and valorise wastes from coffee industry. Moreover the coffee oil presented promising characteristics in the improvement of sunscreen performance.

\section{Acknowledgments}

This work was supported by the Fundação para a Ciência e a Tecnologia, Portugal (UID/DTP/04138/2013 to iMed.ULisboa and grant SFRH/BDE/51599/2011), the strategic project UID/QUI/50006/2013 and Laboratórios Atral S.A., Portugal.

\section{References}

Ahn, S., Yang, H., Lee, H., Moon, S., Chang, I., 2008. Alternative evaluation method in vitro for the water-resistant effect of sunscreen products. Skin Res. Technol. $14,187-191$

Alves, R., Casal, S., Oliveira, M., Ferreira, M., 2003. Contribution of FA profile obtained by high-resolution GC/chemometric techniques to the authenticity of green and roasted coffee varieties. J. Amer. Oil Chem. Soc. 80, 511-517.

Ascenso, A., Ribeiro, H., Marques, H.C., Oliveira, H., Santos, C., Simões, S., 2014. Tretinoin still as a key agent for uv photoaging management. Mini Rev. Med. Chem. 14, 629-641.

Brunner, G., 2013. Gas Extraction: An Introduction to Fundamentals of Supercritical Fluids and the Application to Separation Processes. Springer Science \& Business Media

BS, 1993. Methods for Determination of Particle Size Distribution. Part 4, Guide to Microscope and Image Analysis Methods. British Standards, pp. 36, 3406-4.

Budryn, G., Nebesny, E., Rachwał-Rosiak, D., 2013. Stability of hydroxycinnamic acids and caffeine from green coffee extracts after heating in food model systems. Eur. Food Res. Technol. 236, 969-978.
Calixto, F., Fernandes, J., Couto, R., Hernandez, E.J., Najdanovic-Visak, V., Simoes, P.C., 2011. Synthesis of fatty acid methyl estersvia direct transesterification with methanol/carbon dioxide mixtures from spent coffee grounds feedstock. Green Chem. 13, 1196-1202.

Chiari, B.G., Trovattib, E., Pecorarob, E., Corrêaa, M.A., Cicarellia, R.M., Ribeiro, S., Isaac, V., 2014. Synergistic effect of green coffee oil and synthetic sunscreen forhealth care application. Ind. Crops Prod. 52, 389-393.

COLIPA, 2005. Guidelines For Evaluating Sun Product Water Resistance.

COSMOS, 2013. Cosmetics organic and natural standard. COSMOS-standard AISBL, Brussels, pp. 1-30.

Couteau, C., Deme, A., Cheignon, C., Coiffard, L.J., 2012. Influence of the hydrophilic-lipophilic balance of sunscreen emulsions on their water resistance property. Drug Dev. Ind. Pharm. 38, 1405-1407.

Couto, R., Fernandes, J., Silva, M., Simões, P., 2009. Supercritical fluid extraction of lipids from spent coffee grounds. J. Supercrit. Fluids 51, 159-166.

Dai, J., Mumper, R., 2010. Plant phenolics: extraction, analysis and their antioxidant and anticancer properties. Molecules (Basel, Switzerland) 15, 7313-7352.

Draelos, Z.D., 2011. Cosmetic Dermatology: Products and Procedures. Wiley.

Dutra, E., Oliveira, D., Kedor-Hackmann, E., Santoro, M., 2004. Determination of sun protection factor (SPF) of sunscreens by ultraviolet spectrophotometry. Braz. J. Pharm. Sci. 40, 381-385.

EC, 2009. Regulation (EC) No 1223/2009 of the European Parliament and of the Council of 30 November 2009 on cosmetic products, in: EC (Ed.). Official Journal of the European Union.

Folter, J.W., Ruijven, M.W., Velikov, K.P., 2012. Oil-in-water Pickering emulsions stabilized by colloidal particles from the water-insoluble protein zein. Soft Matter 8, 6807-6815.

Fregert, S., Bandmann, H.J., 1975. International Contact Dermatitis Research Group patch testing. Springer-Verlag, New York, pp. 8-10.

Kale, S., Ghoge, P.P., Ansari, A., Waje, A., Sonawane, A., 2010. Formulation and in-vitro determination of sun protection factor of Nigella sativa Linn. Seed oil sunscreen cream. Int. J. Pharm. Technol. Res. 2

Kelkar, S., Saffron, C.M., Chai, L., Bovee, J., Stuecken, T.R., Garedew, M., Li, Z., Kriegel, R.M., 2015. Pyrolysis of spent coffee grounds using a screw-conveyor reactor. Fuel Process. Technol. 137, 170-178

Laredj-Bourezg, F., Chevalier, Y., Boyron, O., Bolzinger, M.A., 2012. Emulsions stabilized with organic solid particles. Colloids Surf. A 413, 252-259.

Luo, Z., Murray, B., Yusoff, A., Morgan, M., Povey, M., Day, A., 2011. Particle-stabilizing effects of flavonoids at the oil-water interface. J. Agric. Food Chem. 59, 2636-2645.

Mansur, J.S., Breder, M.N.R., Mansur, M.C.A., Azulay, R.D., 1986. Determinação do fator de proteção solar por espectrofotometria. Anais Brasileiros de Dermatologia 61, 167-172.

Marku, D., Wahlgren, M., Rayner, M., Sjöö, M., Timgren, A., 2012. Characterization of starch Pickering emulsions for potential applications in topical formulations. Int. J. Pharm. 428, 1-7.

Marto, J., Gouveia, L., Jorge, I.M., Duarte, A., Gonçalves, L.M., Silva, S.M.C., Antunes, F., Pais, A.A.C.C., Oliveira, E., Almeida, A.J., Ribeiro, H.M., 2015. Starch-based Pickering emulsions for topical drug delivery: a QbD approach. Colloids Surf. B: Biointerfaces 135, 183-192

Marzulli, F.N., Maibach, H.I., 1976. Contact allergy: predictive testing in man. Contact Derm. 2, 1-17.

Pal, R., 1996. Effect of droplet size on the rheology of emulsions. AIChE J. 42, 3181-3190

Preedy, V.R., 2014. Coffee in Health and Disease Prevention. Academic Press. Renner, G., Nano-Titanium Dioxide in Sunscreens, in: COLIPA (Ed.) Brussels 2009.

Ribeiro, H., Marto, J., Raposo, S., Agapito, M., Isaac, V., Chiari, B., Lisboa, P., Paiva, A., Barreiros, S., Simões, P., 2013. From coffee industry waste materials to skin-friendly products with improved skin fat levels. Eur. J. Lipid Sci. Technol. $115,330-336$.

Saewan, N., Jimtaisong, A., 2013. Photoprotection of natural flavonoids. J. Appl. Pharm. Sci. 3, 129-141.

Sayre, R.M., Agin, P.P., Levee, G.J., Marlowe, E., 1979. Comparison of in vivo and in vitro testing of sunscreening formulas. Photochem. Photobiol. 29, 559-566.

Serpone, N., Dondi, D., Albini, A., 2007. Inorganic and organic UV filters: their role and efficacy in sunscreens and suncare products. Inorg. Chim. Acta 360, 794-802.

Shyr, T., Ou-Yang, H., 2015. Sunscreen formulations may serve as additional water barrier on skin surface: a clinical assessment. Int. J. Cosmet. Sci.

Singh, G., Joyce, E.M., Beddow, J., Mason, T.J., 2012. Evaluation of antibacterial activity of $\mathrm{ZnO}$ nanoparticles coated sonochemically onto textile fabrics. J. Microbiol. Biotechnol. Food Sci. 2, 106-120.

Smijs, T.G., Pavel, S., 2011. Titanium dioxide and zinc oxide nanoparticles in sunscreens: focus on their safety and effectiveness. Nanotechnol. Sci. Appl. 12, 95-112.

Speer, K., Kölling-Speer, I., 2006. The lipid fraction of the coffee bean. Braz. J. Plant Physiol. 18, 201-216.

Wagemaker, T., Carvalho, C., Maia, N., Baggio, S., Guerreiro, O., 2011. Sun protection factor, content and composition of lipid fraction of green coffee beans. Ind. Crops Prod. 33, 469-473.

Wang, S.Q., Tooley, R., 2011. Photoprotection in the era of nanotechnology. Semin. Cutan. Med. Surg. J. 30, 210-213. 